\title{
GENIE: LA NIÑA SALVAJE. EL EXPERIMENTO PROHIBIDO (UN CASO DE MALTRATO FAMILIAR Y PROFESIONAL).
}

\author{
AGUSTÍN MOÑIVAS LÁZARO \\ CARMEN SAN CARRIÓN \\ $M^{\mathrm{a}}$ CARMEN RODRÍGUEZ FERNÁNDEZ \\ E.U. Trabajo Social. Universidad Complutense. Madrid.
}

"El maltrato infantil está determinado por fuerzas múltiples que actúan en la familia, en el individuo, en la comunidad y en la la cultura a la que el individuo y la familia pertenecen."

Belsky, 1980 (p. 320)

\section{INTRODUCCIÓN.}

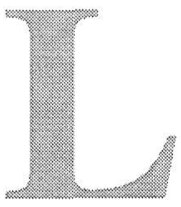

os casos de niños salvajes o que han vivido confinados siempre han provocado una enorme fascinación social. Los historiales o testimonios de casos que precedieron al de Genie han sido muchos y de muy diferente índole (véase Malson, 1973).

Científicamente, todavía no hay acuerdo en cuanto a la polémica naturaleza-cultura; inclusive, en el caso de "el niño salvaje de Aveyron" -el mejor documentado-, se discute sobre si los métodos de aprendizaje empleados fueron los adecuados, dadas las especiales características de Víctor (Itard, 1982; Lane, 1984).

En 1970, y con todos los avances científicos de nuestra época, Genie, una adolescente salvaje, debido a un confinamiento de más de once años, salía de su deprivación. Los trabajadores sociales de una oficina de bienestar de Los Angeles (California) informan a la policía de una madre que se ha presentado junto con su hija en estado de abandono y maltrato. En este caso, al maltrato que ya supone el confinamiento se unían todo tipo de abusos. Al mismo tiempo, dado el subdesarrollo en todas las áreas, la práctica ausencia de lenguaje y el estado de malnutrición evidente, Genie fue remitida al hospital infantil de la zona.

El caso Genie adquirió gran notoriedad debido a dos hechos: uno, científico, y el otro un acontecimiento social. Científicamente, Genie permitía investigar el debate herencia-ambiente (si una buena educa- 
ción y tratamiento permitía compensar una infancia tan terrible) y si hay unos periodos críticos para las emociones y el aprendizaje (en concreto, el establecimiento de los vínculos afectivos o conductas de apego y la adquisición del lenguaje). Debido a ello, el caso Genie se conoce como el "experimento prohibido", ya que ningún ser humano puede ser sometido a tal grado de confinamiento con la finalidad de investigar. En cuanto a acontecimiento social, a las pocas semanas de ser descubierta Genie, se estrenaba en Hollywood "El pequeño salvaje", de J.F. Truffaut; científicos de todo el país fueron invitados a un pase privado.

En este trabajo -reconocida la naturaleza compleja y multifacética de la etiología del maltrato infantil-, desde una perspectiva multidisciplinar e integradora, nos servimos del modelo ecológico-sistémico de Bronfenbrenner (1979) y de los desarrollos de Belsky (1980, 1984, 1993). Metodológicamente, partiendo del análisis documental que disponemos de Genie, organizamos los datos en los diferentes niveles de análisis del modelo, junto al desarrollo ontogenético, para inferir constructos significativos, como la historia de crianza de los padres, experiencias previas en el cuidado de niños, grado de conflictos de la pareja, interacción padres-niño, desestructuración familiar, estrés ocupacional, características del niño que influyen como variable en el maltrato y la amplitud de la asistencia que proveen sus redes sociales de pertenencia.

\section{HISTORIA SOCIAL.}

El 4 de noviembre de 1970 unos asistentes sociales de Los Angeles (California) descubren un extraño caso de una niña de tan sólo trece años que había estado prácticamente toda su vida aislada del mundo. En un aprimera evaluación de su estado, presenta los siguientes síntomas:

- Subdesarrollo en todas las áreas .

- Ausencia de lenguaje.

- Malnutrición.

- Evidentes síntomas de maltrato y abandono.

Para entender este caso, primero debemos describir y evaluar el entorno familiar de Genie. Su madre, al casarse, dice «mi vida ha muerto». Su marido trató de matarla.

Al padre de Genie no le gustaban los niños. Tras cinco años de matrimonio, su madre quedó embarazada y el padre intentó estrangularla otra vez. Nació el bebé y el padre no pudo soportarlo, hasta tal punto que la encerró en el garaje para no sentirle llorar. A los dos 
meses y medio el bebé moriría de neumonía.

Al siguiente año, nacería otro niño, que murió tan sólo dos días después. Se alegó que tragó sus propias mucosidades.

Tres años después, otro hijo nació también con los mismos problemas sanguíneos que el bebé anterior: un $\mathrm{RH}$ incompatible que, en este caso, se mantuvo en un aparente buen estado de salud con el paso de los días. Más adelante, el niño comenzaría a manifestar problemas para aprender a andar, hablar o incluso ir al baño. La abuela paterna se lo llevó a su casa para criarlo, para volver más adelante con sus padres.

Tres años después, en abril de 1957, Genie (cuarto hijo) nacería con un RH incompatible y con la necesidad de una transfusión un día después de nacer. A los tres meses, el pediatra le diagnosticaba una dislocación congénita de la cadera y necesitaría de una prótesis para sostener ambas piernas. Necesitaba, por tanto, fisioterapia, pero el padre se negó a ello e incluso a que su madre la atendiese. A los once meses tan sólo pesaba 17 libras. A los catorce meses, con fuertes fiebres, le fue diagnosticada una neumonía.

Todos estos problemas tuvieron nefastas consecuencias para Genie, ya que, antes de los veinte meses de edad, quedó encerrada en una pequeña habitación, atada de día a una silla y en un cuna cerrada con rejilla metálica de noche. Desde la habitación no podía ver la calle, ni tampoco ningún elemento interior, ya que estaba vacía; tan sólo sus ataduras y su cuerpo serían su fuente primaria de visión y tacto. Viviría en esta situación hasta los 13 años y siete meses.

El padre no permitía pasar al cuarto, controlando que nadie en la casa hiciese ruido o entablarse una conversación para que Genie no oyese nada. Genie se encargaría de llamar la atención por medio de ruidos que el padre paraba dando fuertes golpes contra la pared de la habitación e, incluso, llegó a imitar a un «perro salvaje», ladrando, sacando dientes y arañando para asustarla, pegándole después.

Al principio, la madre intentaba pasar un rato con Genie cada día, pero fue perdiendo progresivamente la vista, siéndole difícil darle algún cuidado. Su hermano, entonces, sería su principal cuidador, pero el padre se lo prohibiría, obligándole a asustarla actuando, también, como un perro salvaje.

Su dieta era estricta. De ello se encargaba el padre. Tan sólo tomaba comida de bebé, se atragantaba y escupía la comida y su padre se la restregaba por la cara. Genie, creció en estas penosas condiciones. Cuando tenía doce años, el padre estaba convencido de que moriría pronto. Fue, entonces, cuando la madre buscaría la ayuda de sus padres.

Consiguieron escapar y se quedaron allí. A la tercera semana, la 
madre de Genie iría a la oficina de bienestar. El trabajador social que les atendió llamó rápidamente a su jefe. Se entrevistó a la madre, y lo que vieron y oyeron hizo que llamasen a la policía, tomando a Genie bajo su custodia. Ese día, el padre se suicidó. Dejaría una nota diciendo que «el mundo nunca lo entendería». Genie sería admitida en el hospital por su extrema malnutrición

Noviembre 1970 - enero 1971.

Genie daba pena. Como casi nunca había llevado ropa, no reaccionaba a la temperatura, fuese frío o calor. Nunca había comido nada sólido, por lo que no sabía masticar y le costaba mucho tragar. Por haber estado tanto tiempo atada a la «silla-orinal», Genie no tenía fuerza en las piernas, por lo que no podía correr, subir escaleras, agacharse..., de hecho, sólo podía andar y con mucha dificultad. Como nunca había mirado a una distancia mayor de tres metros, tenía gran dificultad para enfocar a mayores distancias. Como le pegaban por hacer ruido, había aprendido a suprimir cualquier tipo de vocalización. Sufría malnutrición, pesaba sólo 59 libras y medía sólo 54 pulgadas. Padecía enuresis y encopresis, tenía el pelo descuidado y estropeado, salivaba copiosamente y escupía sobre cualquier cosa que tuviese a mano. Carecía de socialización alguna, era primitiva y casi inhumana.

Genie utilizaba su cuerpo y objetos para hacer ruido y ayudarse a expresar su frenesí: arañaba el suelo con una silla, sus dedos rascaban los globos, volcaba los muebles, lanzaba objetos y los golpeaba, arrastraba los pies. Genie se vio enfrentada a la tarea de aprender a hablar. Aunque fuesen verdad las declaraciones de su madre de que había aprendido vocabulario siendo un bebé, era evidente que su entorno no le proporcionó suficientes signos lingüísticos. Sin embargo, era necesario determinar si su cerebro estaba dañado y tenía el necesario desarrollo cognitivo como para adquirir el lenguaje.

\section{Enero1971 - junio 1971.}

El pronóstico de crecimiento era pobre en todas las áreas. Se discutía si su deficiencia era congénita o fruto de la deprivación. A pesar de ello, Genie, empezó a progresar en todas las áreas.

Junio 1971-agosto 1971.

Susan Curtiss (1977), nuestra principal fuente de información, manifiesta que conoció a Genie en junio del 71: "era muy pequeña, tan pequeña, tan delgada, tan diferente de una chica normal de catorce años... Tenía un comportamiento desagradable, seguía salivando y es- 
cupiendo a todo y todos los que estuviesen a su alcance. También, presentaba comportamientos sociales muy poco aceptables. Se sonaba la nariz en cualquier cosa, a menudo ensuciando su ropa. Cuando se ponía nerviosa se ponía a orinar en cualquier sitio, dejando al «cuidador» a cargo de las consecuencias. Cuando salía de paseo y algo le llamaba la atención, se acercaba al objeto o la persona y se agarraba sin soltarse hasta conseguir lo que deseaba, especialmente los objetos de plástico. No atendía a ninguna distancia social, se acercaba a las personas en las que se fijaba, y les daba la mano o les agarraba del brazo para acompañarles en su paseo.

Genie se masturbaba excesivamente, lo que resultó ser el comportamiento antisocial más problemático de todos; a pesar de las reprimendas, seguía masturbándose en cualquier sitio. No sabemos si su padre o su hermano abusaron sexualmente de ella, pero lo cierto era que prefería estar acompañada de un hombre, intentando establecer relaciones. Aprender a controlar estos deseos, inclusive ahora, cuatro años más tarde, sigue siendo un problema.

Genie tenía miedo al perro. Se asustaba cuando sentía que podía acercarse, huía de él subiéndose a las mesas, sillas, corriendo..., intentando poner alguna barrera entre ella y el perro. Sus movimientos se volvían más rápidos y era más ágil cuando sentía al perro, lo que choca con el hecho de que normalmente sus movimientos eran muy lentos y actuaba despacio.

Septiembre 1971-1975 (evaluaciones y notas de Curtiss hasta 1977).

- Respuestas retardadas:

Cuando a Genie se le formulaban preguntas o se le pedía que hiciese algo, reaccionaba como si nadie le hubiese hablado. Tan sólo, pasados unos minutos, Genie contestaba a la pregunta o actuaba conforme a lo que se le pidió, aunque hubiesen sucedido cosas o intercalado conversaciones.

- Comportamiento perezoso:

Genie elegía los modos de actuación que le exigían menor esfuerzo. Delegaba en los demás actividades para las que ella era capaz. Trataba de realizar el mínimo esfuerzo. Lo mismo ocurría con el lenguaje: omitía frases o palabras que podía pronunciar, tan sólo expresaba lo que le resultase estrictamente necesario y con una o dos sílabas y señalando el objeto. Llegó a adquirir un «comportamiento ritual».

- Uso de gestos y aprendizaje del habla:

Dada su dificultad para hablar, al principio de su aprendizaje, usaba más a menudo expresiones faciales o corporales para comunicarse, 
inventando su propio repertorio de vocabulario gestual. En octubre de 1971, se evidenciaba que Genie empezaba a ser receptiva a las diversas situaciones lingüísticas de su entorno. En enero de 1972, Genie comenzó a utilizar el lenguaje por primera vez para referirse a un acontecimiento pasado. Genie aprendió a usar el lenguaje para mentir y encubrir la realidad de lo que hubiese sucedido, incluso para hablar acerca de sus fantasías sexuales. No obstante, no lograba vocalizar correctamente por haber estado tantos años reprimida de hacerlo.

- Afecto y desarrollo social:

Como Genie ha aprendido a expresarse de manera más completa, ha desarrollado un mejor concepto de ella misma y ha aprendido a expresar y controlar sus sentimientos.

- Entrevistas con la madre de Genie:

El trabajador social del hospital realizó entrevistas a la madre de Genie acerca de la vida de ésta antes de ser descubierta. Los informes de las entrevistas no eran fiables, ya que descubrimos que la madre a menudo contestaba en función de lo que ella pensaba que el entrevistador quería oír. Además, se contradecía a menudo de una entrevista para otra. Sólo a través de la observación del comportamiento de Genie pudimos descubrir algunos detalles de su terrible pasado.

- Informes del Departamento de Psiquiatría:

El caso de Genie tiene una implicación directa con la hipótesis de la existencia de un «periodo crítico» para la adquisición del lenguaje. Lenneberg en 1967 formula la existencia del «periodo crítico» para el desarrollo del lenguaje humano que va desde los dos años hasta la pubertad. Este periodo crítico necesita dos condiciones: tener un cerebro humano (lo que implica socialización, comunicación y afectividad desde el nacimiento -Bolwy; cfr. Howe, 1996-) y una estimulación lingüística.

Como resumen de su historial en el Hospital, podemos decir que el equipo de investigación no consiguió definir una línea de investigación coherente para Genie, primando a Genie como objeto de investigación y no como ser humano (de hecho, algunos investigadores la adoptaron con fines partidistas). El caso acabó siendo denunciado, a través de la madre, las subvenciones a la investigación retiradas y todos los principales investigadores encausados. Tras abandonar el hospital, Genie tuvo al menos seis hogares adoptivos, en algunos de ellos con maltrato. Actualmente, vive en un centro de acogida para personas mayores en Los Angeles. Genie cumplirá 45 años el próximo mes de abril. 


\section{CONSIDERACIONES TEÓRICAS.}

Los malos tratos han sido abordados desde áreas legales, médicas, psicológicas, asistenciales, etc. Estás diferentes ópticas nos permiten explicar la diversidad de definiciones y la necesidad de un enfoque multidisciplinar e integrador.

La definición de los malos tratos debe considerar la heterogeneidad del fenómeno, atendiendo a las necesidades físicas y psicológicas del niño y a los diferentes contextos de desarrollo: familia, guardería, escuela, vecindario, comunidad, cultura, etc., para la satisfacción de las mismas.

La interacción necesidades-contexto provee toda una serie de abandonos y abusos que pueden definirse a partir de tres factores: contexto-familiar-extrafamiliar, formas activas-pasivas y aspectos emocionales o físicos afectados.

Otro tres factores que dan cuenta del ser humano al nacer, y de los que Genie careció, son: la incapacidad de sobrevivir por sí mismo, la necesidad del contacto físico y de establecer vínculos afectivos o sociales y una manera de interacción con el entorno que permite la asimilación o acomodación de la realidad, según Piaget.

El primer contexto responsable de la supervivencia del niño es el familiar. En dicho contexto el niño ha de satisfacer las necesidades primarias físicas y socioemocionales de las que nos habla Maslow. Genie careció de ambas en el contexto familiar, y de la emocionales -vínculos afectivos seguros- en el hospital, por la diversidad de profesionales implicados en su caso.

Desde este presupuesto, el maltrato es cualquier acción persisente, por parte de los padres, cuidadores o profesionales, que compromete la satisfacción de las necesidades básicas. Genie fue objeto, desde el punto de vista físico, de abusos físicos y sexuales de una manera activa, y de abandono físico de una manera pasiva. Desde un punto de vista emocional, Genie sufrió maltrato emocional activo y abandono emocional pasivo.

\section{DEFINICIONES OPERACIONALES.}

Operacionalizando los términos empleados, y siguiendo a De Paúl et al. (1988), que aplica esta misma metodología a la identificación de factores de riesgo en la infancia en el País Vasco, distinguimos entre abusos y abandonos físicos y emocionales. Podemos definir el abuso físico como cualquier acción, no accidental, por parte de los padres o 
cuidadores que provoque daño físico o enfermedad en el niño; y el abuso sexual como cualquier clase de contacto sexual con un niño menor de 18 años por parte de un familiar/tutor adulto desde una posición de poder o autoridad sobre el niño. Por su parte, el abandono físico implica que las necesidades físicas de alimentación, vestido, higiene, protección y vigilancia, así como las atenciones médicas, no son atendidas por ningún miembro del grupo que convive con el niño (índices de este tipo de maltrato son: retraso en el crecimiento, enfermedades o problemas médicos no tratados, malnutrición, falta de higiene, vestido inadecuado, falta de escolarización...); y el abandono emocional como la falta persistente de respuesta a las señales (llanto, sonrisa), expresiones emocionales y conductas procuradoras de proximidad e interacción de proximidad e interacción iniciadas por el niño y falta de iniciativa y contacto por parte de una figura estable durante los seis primeros años de vida. Indicadores físicos y comportamentales de este tipo de abuso son: retraso en el crecimiento, retraso intelectual y del lenguaje, falta de expresividad, pasividad, tristeza, apatía, indefensión, dificultades para establecer relaciones sociales. En cuanto al abuso emocional podemos definirlo como cualquier forma de insulto, burla, desprecio, crítica o amenaza de abandono y constante bloqueo de las iniciativas infantiles (desde la evitación hasta el encierro o confinamiento) por parte de cualquier miembro de la familia.

En Genie estos tipos de abusos y abandonos son crónicos, viviendo con ellos hasta el inicio de la adolescencia en el contexto familiar, y posteriormente en alguna forma de abuso emocional en el hospital, con los miembros del equipo investigador, o, inclusive, en casa de alguno de ellos, al ser más objeto de investigación que de tratamiento.

Desde esta perspectiva, nos parece que el modelo ecológico-sistémico de Bronfenbrenner (1979) y los desarrollos de Belsky (1980, 1984, 1993), a partir de dicho modelo, dan perfecta cuenta de este caso.

En concreto, y para integrar el historial de Genie, tenemos en cuenta los subsistemas micro, meso, exo y macro de Bonfrenbrenner (Moñivas, 1998) y el desarrollo ontogenético aportado por Belsky. En el microsistema, anillo del anillo del niño, tenemos en cuenta las características personales, inclusive las que pueden elicitar maltrato, aunque no justificarlo, según diferentes autores (temperamento, temprano desarrollo ontogenético); en el mesosistema, anillo de la familia, tenemos en cuenta las características y habilidades de los padres, del niño, la interacción madre-padre-niño y el conflicto de pareja; en el exosistema, el trabajo, los vecinos y la clase social; y el macrosistema, las representaciones sociales sobre la infancia, la familia, la mujer, la violencia y el castigo físico (Moñivas, 1992). Con respecto al desarrollo ontogenético, tenemos en cuenta la posible historia de malos tratos de los pa- 
dres. Todo este análisis puede verse en las tablas, diagramas y cuadros que se expondrán en el Congreso. Los dos contextos principales de análisis han sido la familia y el hospital.

\section{CONCLUSIONES.}

Aunque el caso de Genie es extremo, permite poner de manifiesto, por la magnitud de todas las conductas implicadas, que toda intervención sobre la violencia debe atender a las necesidades físicas y psicológicas del sujeto y a los diferentes contextos para la satisfacción de las mismas, considerando los factores que permiten definir el maltrato, que tienen lugar en la interacción necesidades-contexto, y las características del ser humano al nacer y en su desarrollo, así como evaluar las redes sociales de apoyo de toda familia en el momento de la crianza o la actuación desde los Servicios Sociales en cuanto a la prevención y educación socio-familiar.

Desde la investigación, podemos decir que la comprensión del maltrato infantil, así como su capacidad predictiva y preventiva irán en aumento a medida que la interacción entre las variables de los diferentes niveles esté bien establecida y analizada (véase: Ward y Finkeldors, 2000).

\section{BIBLIOGRAFÍA.}

BELSKY, J.: "Child maltreatment. An Ecological Integration". American Psychologist, 35 (4), 320-335, 1980.

BELSKY, J.: "The determinants of parenting: A Process Model". Child development, 35(4), 1984.

BELSKY, J.: "Etiology of child maltreatment. A developmental-ecological analysis". Psychological Bulletin, 114 (3), 320-335, 1993.

BRONFENBRENNER, U.: La Ecología del desarrollo humano. Paidós, Barcelona, 1979.

CURTISS, S.: A psycholinguistic study of a Modern-Day "Wild Child. Academic Press, New York, 1977.

DE ANDA, D. \& BECERRA, R. (eds.): Violence. Diverse populations and communities. The Harworth Press, New York, 2000.

DE PAÚL OCHOTORENA, J. (dir.): Maltrato y abandono infantil. Factores de riesgo. Servicio de Publicaciones del gobierno vasco, Vitoria-Gasteiz, 1988.

ITARD, J.: Memoria e informe sobre el niño salvaje del Aveyron. Comentarios de Rafael Sánchez Ferlosio. Alianza, Madrid, 1982. 
JONES, P.: Contradictions and unanswered questions in the Genie case: A fresh look at the linguistic evidence. Language-and-Communication, 15(3), 261-280, 1995.

LANE, H.: El niño salvaje de Aveyron". Alianza Universidad, Madrid, 1984.

MARTÍNEZ ROIG, A.: Factores de vulnerabilidad infantil para el maltrato y abandono. En J. de Paúl (dir.), El maltrato y abandono infantil. Servicio de Publicaciones de la Universidad del País Vasco, Lejona (Vizcaya), 1987.

MOÑIVAS, A.: "Representación e intervención social". Cuadernos de Trabajo Social, Escuela de Trabajo Social de la UCM, Madrid, 4-5,69-88.

MOÑIVAS, A.: "Multiculturalidad: Ciencia y Sociedad". Servicios Sociales y Política Social, Consejo General de Colegios Oficiales de Diplomados en Trabajo Social, Madrid, 1998,44, 9-32.

RYMER, R.: Genie: An abused child's flight from silence. Harper Collins Publishers, New York, 1993.

SKUSE, D.: "Extreme deprivation in early chidhood. Theoretical issues and a comparative review". Journal of Child Psychology, 25(4), 542-72, 1984.

WARD, S. \& FINKELDORS, D. (eds.): Program Evaluation and Family Violence Research. The Harworth Press, New York, 2000.

A continuación se complementa la bibliografía con la posibilidad de consultar la siguiente videorreferencia:

- "No estamos locos".

- A Child's First words: by Piaget.

- Child's Play.

- Película "El niño salvaje de Aveyron" de Truffaut.

- Película "El secreto de Caspar Hauser" de Werner Herzog.

- Erquicia, P.: "Genie: La Niña Salvaje". Documentos TVE, Madrid.

Páginas web donde se ofrece información:

www.psicoactiva.com

www.ciudadfutura.com

http://membersfortunecity.com 